\title{
Phorbol Diesters Stimulate the Development of an Early Murine Progenitor Cell
}

\author{
The Burst-forming Unit-Megakaryocyte \\ Michael W. Long, Linda L. Gragowski, Connie H. Heffner, and Laurence A. Boxer \\ Division of Hematology/Oncology, Department of Pediatrics, University of Michigan, Ann Arbor, Michigan 48109
}

\begin{abstract}
When murine (C57BL/6) bone marrow cells are cultivated with WEHI-3 conditioned media, a source of megakaryocytecolony-stimulating activity (Mk-CSA), and phorbol myristate acetate (PMA), a previously undetected population of megakaryocyte (Mk) progenitor cells is observed. These new Mk colonies are reminiscent of erythroid bursts, in that they contain large numbers (40-500) of $\mathrm{Mk}$ and multiple foci (27) of development. These burst-forming units, Mk (BFU-Mk), are defined as having $\geq 42$ cells/colony and, at least, three foci of Mk development (colonies grown in soft agar cultures, all studies done at limiting dilutions; colonies detected by acetylcholinesterase [ACh-E] staining). CFU-Mk and BFU-Mk require two activities for optimal growth: Mk-CSA and PMA. However, the BFU-Mk require a tenfold greater concentration of PMA for optimal development $\left(10^{-6} \mathrm{vs} .10^{-7} \mathrm{M}\right)$. BFU-Mk detection is linear (over a range of $25-100 \times 10^{3}$ cells $/ \mathrm{ml}$ ), with the regression line passing through the origin. Bone marrow frequencies of these two progenitor cells are CFUMk, 36.7 \pm 2.5 , and BFU-Mk, 7.3 \pm 0.7 per $10^{5}$ total nucleated cells (mean \pm SEM; $n=28$ ). The BFU-Mk have a restricted velocity sedimentation range $\left(3.3-4.5 \mathrm{mmh}^{-1}\right.$ vs. $3.3-6.8 \mathrm{mmh}^{-1}$ for CFU-Mk). Modal buoyant densities are $1.068 \pm 0.0002$ and $1.070 \pm 0.002$ for BFU-Mk and CFU-Mk, respectively. Thus, these cells are found among the smallest and less dense of the Mk progenitors, and are not clumps or clusters of CFU-Mk. Kinetic analysis indicates that CFU-Mk require 5-7 d for optimal growth, whereas BFU-Mk require 10-12 d. Examination of the proliferative potential (cells per colony) shows $19.3 \pm 1.5$ cells per colony ( $n=246$ colonies) for day 10 CFUMk, vs. $118 \pm 6.0$ for day 10 BFU-Mk $(n=163)$. Analysis of the cellularity/subcolony within each burst indicates $37.0 \pm 2.1$ $(n=146) \quad M k / c o l o n y$ and $3.9 \pm 0.1$ subcolonies/burst ( $n$ $=100)$. Finally, $>90 \%$ of the BFU-Mk contain only ACh-E positive cells, indicating that these are not mixed colonies. These results indicate that the BFU-Mk, compared with the CFU-Mk, require an increased amount of stimulation in order to differentiate, show delayed in vitro development, and have a higher proliferative potential. These data are consistent with the hypothesis that these cells are early progenitor cells in the Mk lineage that antedate the CFU-Mk.
\end{abstract}

This paper was presented in part at the 24th Annual Meeting of the American Society of Hematology, 1984.

Dr. Long is a Scholar of the Leukemia Society of America.

Received for publication 21 December 1984 and in revised form 3 April 1985

J. Clin. Invest.

(c) The American Society for Clinical Investigation, Inc.

0021-9738/85/08/0431/08 $\$ 1.00$

Volume 76, August 1985, 431-438

\section{Introduction}

In contrast to the later colony-forming units (CFU), ${ }^{1}$ early committed progenitor cells can be characterized by a high proliferative capacity, altered in vitro requirements for regulatory activities, and distinctly different rates of colony development. Such early progenitor cells are exemplified by the erythroid burst-forming unit (BFUe) and the macrophage high proliferative potential colony-forming cell (HPP-CFC) (1-4). Previous reports have not documented the presence of similar cells in the megakaryocytic (Mk) lineage (5-9).

We have shown that tumor-promoting phorbol diesters can substitute for poorly defined biological activities known to influence megakaryocyte colony-forming cells (CFU-Mk) (10). The use of these well-characterized compounds allows precise control of the degree of in vitro Mk development. Thus, phorbol diesters serve as powerful probes of the cellular mechanisms governing Mk differentiation. When murine bone marrow cells are co-cultivated with $4 \beta$ phorbol 12-myristate 13-acetate (PMA) and WEHI-3 conditioned medium (CM), a source of Mk colony-stimulating activity (Mk-CSA), a previously undetected population of Mk progenitor cells, is observed. These Mk colonies are reminiscent of BFUe, in that they form colonies that contain large numbers of $\mathrm{Mk}$ and have multiple foci of individual colony development. We hypothesize that these burst-forming unit Mk (BFU-Mk) are early hematopoietic progenitor cells which are restricted to the $\mathrm{Mk}$ lineage and are characterized by a high degree of proliferative potential.

\section{Methods}

$M k$ progenitor cell assays (CFU-Mk and BFU-Mk). Cells from both femurs of male mice (C57BL/6 Charles River Breeding Laboratories, Wilmington, MA), 6-10-wk-old, were flushed into McCoy's 5A medium and monodispersed. Before plating, the cell suspension was incubated at $37^{\circ} \mathrm{C}$ for $30-60 \mathrm{~min}$ at $\sim 3-5 \times 10^{6}$ cells $/ \mathrm{ml}$ in McCoy's $5 \mathrm{~A}$ medium containing 5-10\% FCS. Marrow cells were cultured at various concentrations in 35-mm Petri dishes (Falcon 3001) in modified McCoy's 5A medium containing $10 \%$ FCS, $0.25 \%$ Bacto-agar (Difco Laboratories, Detroit, MI), and $3 \times 10^{-7} \mathrm{M}$ prostaglandin E2 (Sigma Chemical Co., St. Louis, MO) (11). Bone marrow cells were co-cultured with CM from a murine monomyelocytic cell line (WEHI-3), plus mouse lung $\mathrm{CM}(11)$ or PMA (10). After incubation $\left(7 \mathrm{~d}\right.$ at $37^{\circ} \mathrm{C}, 7 \% \mathrm{CO}_{2}$ and $100 \%$ humidity), petri dishes were removed from the incubator, dried, and stained in situ for acetylcholinesterase (ACh-E) activity (12). Plasma-clot cultures were supplemented as above, substituting $10 \%$

1. Abbreviations used in this paper: BFU-Mk, burst-forming unit megakaryocyte(s); BFUe, BFU erythrocyte(s); CFU, colony-forming unit(s); CM, conditioned medium; HPP-CFC, high proliferative potential colony-forming cell; Mk-CFUs, megakaryocyte CFU spleen; Mk-CSA, Mk colony-stimulating activity; MLuCM, murine lung CM; PMA, $4 \beta$ phorbol 12-myristate 13-acetate. 
bovine plasma (HyClone Inc., Logan, UT) for Bacto-Agar. Erythropoietin added to these cultures was either sheep, step III (Connaught), or purified murine erythropoietin (HyClone Inc.). These plasma-clot cultures supported equivalent numbers of colonies compared with agar cultures. Mk colonies were enumerated at $\mathbf{4 0}$ magnifications. CFUMk were functionally defined as a cell capable of giving rise to $>3$ $\mathrm{Mk}$ /colony. BFU-Mk were similarly defined as a cell generating $\geq 42$ cells/colony and at least three foci of Mk development (see below). Examination of the cellularity (cells per colony) of a given colony or burst was performed using a disection microscope (Olympus Corp. of America) at 60-120 magnifications. In colonies or bursts of $>50-70$ cells, individual Mk were often greatly overlapped. Thus, only clearly distinct Mk were counted. Therefore, the number of cells per colony in these instances was a conservative estimate of the actual colony cellularity.

Conditioned medium. WEHI-3 cells have been adapted to grow in suspension culture (13). Cells were grown in McCoy's 5A containing $5 \times 10^{-5} \mathrm{M} 2$-mercaptoethanol and $2 \%$ fetal calf serum. The medium was harvested every 3-4 $d$ and concentrated fivefold by ultrafiltration (Amicon YM-10, Amicon Corp., Lexington, MA). Mouse lung CM (MLuCM) was prepared by coarsely cutting murine lungs and suspending one lung per $5 \mathrm{ml} \mathrm{McCoy's} \mathrm{5A} \mathrm{medium.} \mathrm{CM} \mathrm{were} \mathrm{collected}$ after 3 to $4 \mathrm{~d}$ of incubation. Optimal concentrations of these CMs were determined using reciprocal titrations (14). The concentrations used for these studies were: WEHI-3 CM, 5\%; MLuCM, 7.5\% (final concentration).

Phorbol myristate acetate. PMA (Sigma Chemical Co.) was prepared as stock solutions of $10^{-5} \mathrm{M}$ in dimethylsulfoxide and kept in the dark at $-20^{\circ} \mathrm{C}$ until used. Fresh stock solutions were prepared every 3 wk. Immediately before use, a working solution was prepared using McCoy's $5 \mathrm{~A}$ medium as the diluent (10).

Cell separation. Velocity sedimentation was performed at unit gravity according to the method of Miller and Phillips (15). Bone marrow was obtained from 15 to 20 mice by flushing with cold $\left(4^{\circ} \mathrm{C}\right)$ phosphate-buffered saline (PBS). Cells were kept on ice during collection and the separation was carried at $4^{\circ} \mathrm{C}$. After sedimentation for 3-4 h, $35-\mathrm{ml}$ fractions were collected and the cells harvested by centrifugation at $400 \mathrm{~g}$ for $7 \mathrm{~min}$. These cells were resuspended in McCoy's 5A media. The cells were then cultured for CFU-Mk and BFU-Mk content. Total cell recovery in these procedures was $60-85 \%$.

Continuous density gradients (total volume $15 \mathrm{ml}$ ) were generated using Percoll (Pharmacia Fine Chemicals, Piscataway, NJ). The gradient range was $1.050-1.100 \mathrm{~g} / \mathrm{cm}^{3}$ of Percoll suspended in murine tonicity PBS, $\mathrm{pH}$ 7.0. The gradients were formed as follows: equal volumes of low density $\left(1.050 \mathrm{~g} / \mathrm{cm}^{3}\right)$ and high density $\left(1.100 \mathrm{~g} / \mathrm{cm}^{3}\right)$ were placed in separate chambers of a two-chamber gradient maker. The gradients were generated at unit gravity over $20 \mathrm{~min}$. During formation, the two Percoll density functions were gently admixed with a magnet stir bar. In order to improve resolution, cells were suspended at $1-2 \times 10^{7} / \mathrm{ml}$ in the heavy density fraction during formation of the gradient (16). Gradients were spun at $400 \mathrm{~g} \times 15 \mathrm{~min}$ at room temperature. Increasing gravity minutes resulted in loss of continuity of the gradient due to the colloidal nature of Percoll. Rebanding experiments indicated that cells separated on these gradients were achieving equilibrium density, and that the resolution of the method was $\pm 0.006 \mathrm{~g} / \mathrm{mm}^{3}$ (resolution data not shown). After separation, fractions of $1.0 \mathrm{ml}$ were collected by bottom displacement using Fluorinert FC40 (Sigma Chemical Co., density $=1.85 \mathrm{~g} / \mathrm{cm}^{3}$ ). An aliquot of each fraction was then removed for density determination. Cells were harvested by dilution and centrifugation (400 $\mathrm{g} \times 7 \mathrm{~min}$ ) and calculated as cell per fraction per density increment to correct for nonlinearity of the gradient (16). Data were normalized as percentage of peak value. Standard curves were prepared correlating refractive index with density using density marker beads (Pharmacia Fine Chemicals) as a density index. All subsequent density gradients were then measured by refractometry. Modal buoyant densities were determined by plotting cumulative cell frequency vs. density-which is known to decrease background noise in these systems (16).

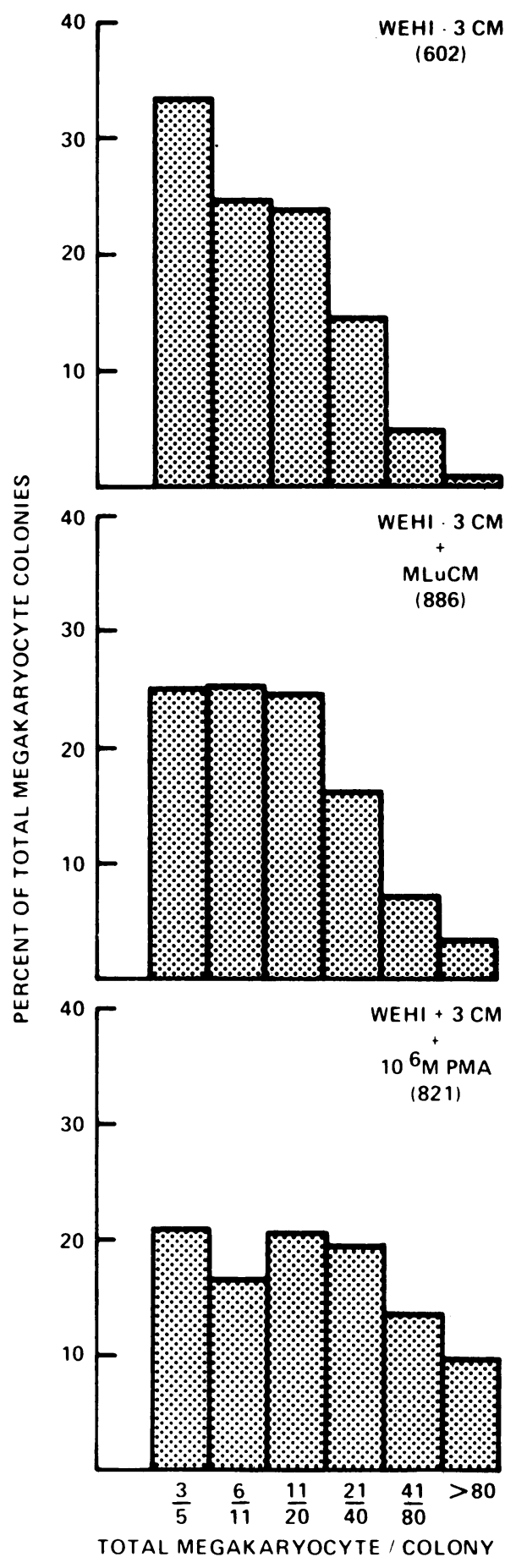

Figure 1. Mk colony cellularity. Mk per colony were counted irrespective of colony morphology. Numbers in parentheses are the total number of colonies counted. (Top) Colonies grown in the presence of WEHI-3 CM only. (Middle) Colonies resulting from co-culture of marrow cells and WEHI-3 CM plus MluCM. (Bottom) Colonies cultivated in the presence of WEHI-3 CM and $10^{-6} \mathrm{M}$ PMA. Numbers on abcissa represent lower and upper levels of incremental colony size, e.g., $3 / 5=3-5 \mathrm{Mk} /$ colony. Colonies cultured at limiting dilutions of $1 \times 10^{5}$ cells $/ \mathrm{ml}$.

\section{Results}

Cellularity of $M k$ progenitor cell colonies. Observations on colony cellularity in phorbol-treated cultures showed a 2.5- 

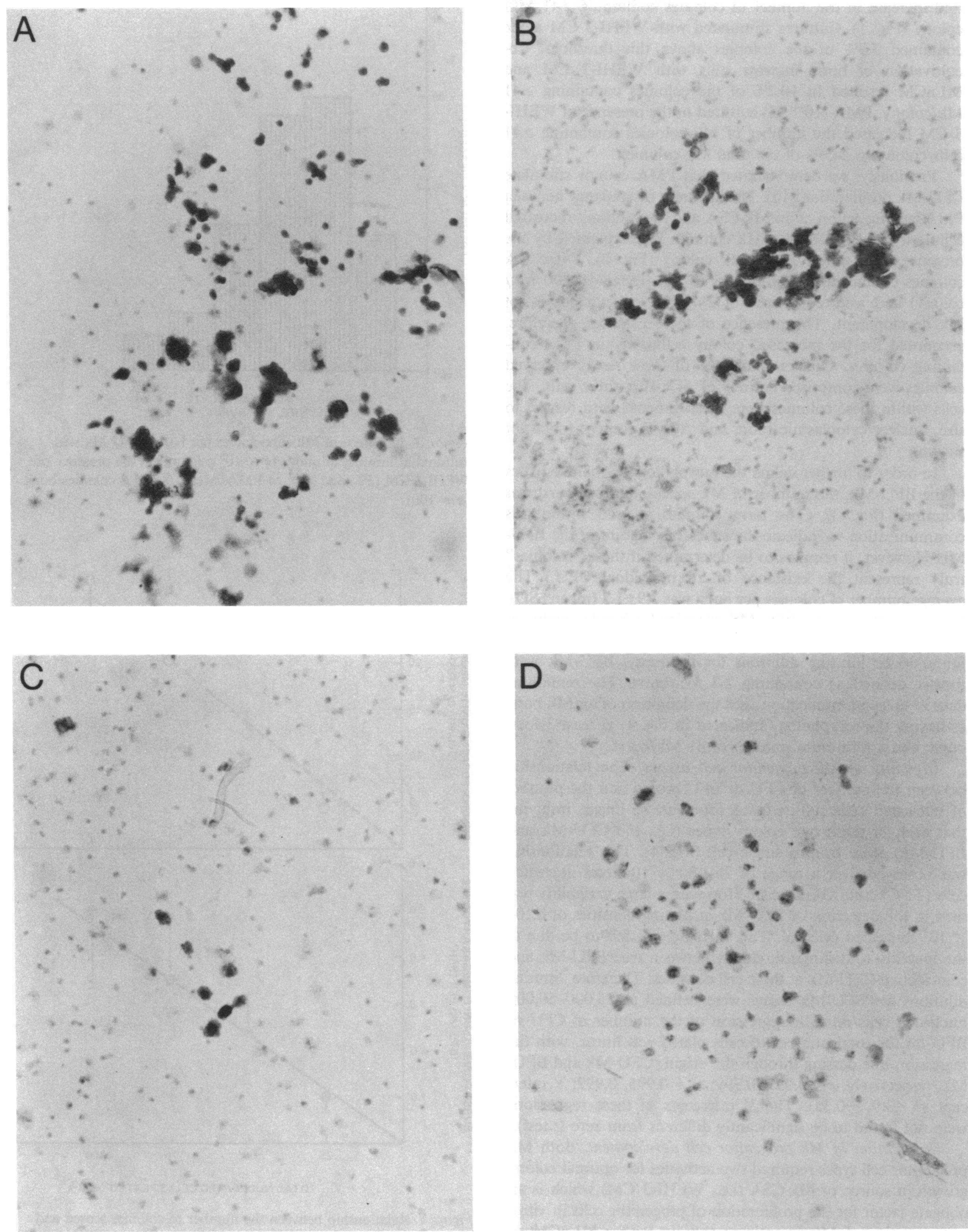

Figure 2. Murine Mk progenitor cells. Colonies were grown in the presence of WEHI-3 CM and $10^{-6} \mathrm{M}$ PMA and stained in situ for ACh-E activity. $(A$ and $B$ ) BFU-Mk colonies (day 10). ( $C$ and $D$ ) CFU-Mk colonies (day 10). 
fold increase in the number of colonies containing $\geq 41 \mathrm{Mk}$ / colony (Fig. 1). Cultures stimulated with WEHI-3 CM alone contained $5.3 \%$ of the colonies above this threshold. Cocultivation of bone marrow cells with WEHI-3 CM and MLuCM resulted in $10.2 \%$ of the colonies containing $\geq 41$ $\mathrm{Mk} /$ colony. PMA $\left(10^{-6} \mathrm{M}\right)$ cultured in the presence of WEHI$3 \mathrm{CM}$ increased the number of $\mathrm{Mk}$ colonies containing $\geq 41$ cells/colony to $24.6 \%$ of the total Mk colonies.

Previously, we demonstrated that PMA cannot stimulate CFU-Mk proliferation (10). The apparent discrepancy between PMA lacking action as an Mk-CSA and the resultant increased cellularity of PMA-driven Mk colonies was explained by the presence of a new type of Mk colony (Fig. 2). These Mk colonies contained large numbers of $\mathrm{Mk}$ (range 41-500 Mk/ colony) and were comprised of 2-8 foci, or subcolonies, of Mk development. The presence of these colonies, therefore, accounted for the increased colony cellularity in PMA-containing cultures. Greater than $90 \%$ of these newly observed colonies were comprised entirely of $\mathrm{ACh}-\mathrm{E}$ positive cells. The cells within these colonies were heterogeneous with respect to size, nuclear/cytoplasmic ratio, and $\mathrm{ACh}-\mathrm{E}$ content (data not shown).

In order to further define the morphological characteristics of the BFU-Mk, the number of Mk subcolonies per burst was examined (Fig. 3). (The term colonies is used within this communication to indicate the subcolony nature of the BFUMk. However, it remains to be determined if these "colonies" truly represent the existence of an individual CFU.) The average number of colonies per burst was $3.9 \pm 1.3$ (mean \pm SD). However, $85 \%$ of the BFU-Mk contained $\geq 3$ colonies/burst. As these original assays were not carried out at what was later found to be limiting dilutions for the assay, BFU-Mk were further defined as containing $\geq 3$ foci/burst. The remaining data in this communication uses the definition of an Mk burst as having the morphology indicated in Fig. 1, at least 3 foci/ burst, and a minimum number of $41 \mathrm{Mk} / \mathrm{burst}$.

Clonality of $M k$ progenitor cell assays. The relationship between the number of CFU or BFU scored and the number of nucleated cells/cultured was found to be linear, implying that each of these two colony types (i.e., the CFU-Mk and BFU-Mk) arose from a single cell (Fig. 4). This relationship was observed over a range of $25-100 \times 10^{3}$ total nucleated cells (TNC) cultured per dish. However, a large variability was seen in the detection of BFU-Mk at plating densities of $\geq 100$ $\times 10^{3}$ nucleated cells $/ \mathrm{ml}$. This increase was felt to be due to the inability to distinguish clearly between true BFU-Mk and coincidental CFU-Mk at these cell densities. Therefore, limiting dilutions for BFU-Mk assays were defined as 25,000-50,000 nucleated cells $/ \mathrm{ml}$. The regression of the number of CFU or BFU on the total number of cells plated was linear, with the regression line passing through the origin (CFU-Mk and BFUMk, respectively, $r=0.997,0.999 ; r^{2}=0.995,0.999 ; Y$ intercept $=-3.9,-0.35)$. The $\mathrm{Y}$ intercepts of these regressions were not found to be significantly different from zero ( $t$ test).

Stimulation of $M k$ progenitor cell development. Both $\mathrm{Mk}$ progenitor cell types required two activities for optimal colony growth: a source of Mk-CSA (i.e., WEHI-3 CM) which is an obligate factor for the proliferation of progenitor cells in vitro, and a source of Mk-potentiator activity (either MLuCM or PMA) which affects Mk differentiation $(10,11)$. The responsiveness of Mk progenitor cells to PMA is shown in Fig. 5. PMA was able to act synergistically with a source of Mk-CSA

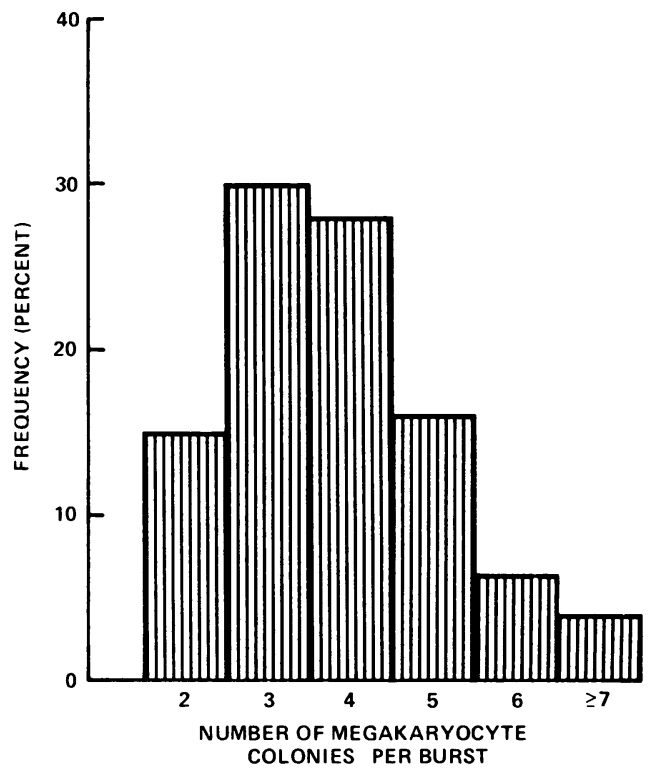

Figure 3. Incidence of Mk subcolonies per burst. BFU-Mk was cultured at limiting dilutions $\left(1 \times 10^{5}\right.$ cells $\left./ \mathrm{ml}\right)$ in the presence of

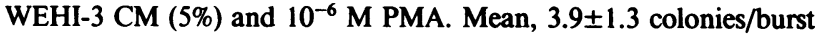
$(n=100)$.
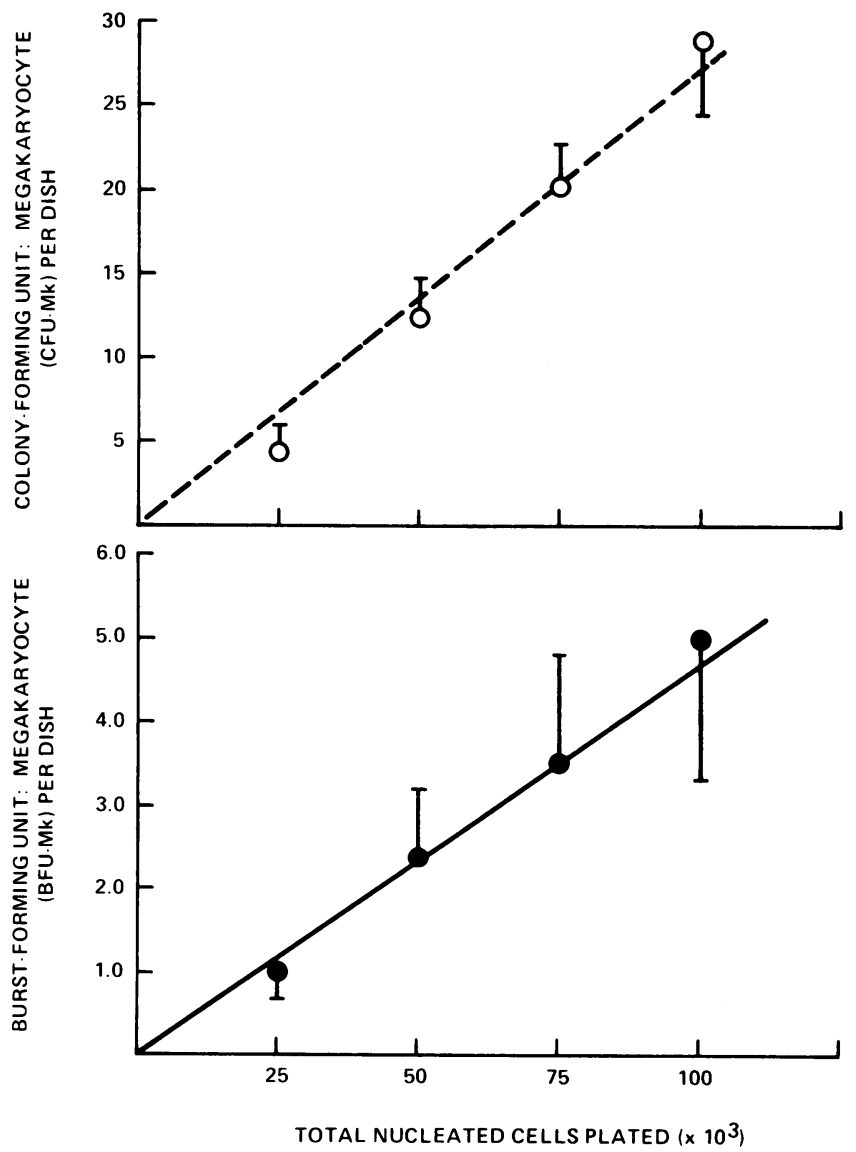

Figure 4. Relationship between the number of colonies scored and the number of nucleated cells cultured. Mk progenitor cells cultured in the presence of WEHI-3 CM (5\%) and $10^{-6} \mathrm{M}$ PMA. Solid circles, BFU-Mk; open circles, CFU-Mk. Values are $\mathrm{X} \pm \mathrm{SD}$ based on six experiments of 4-5 replicate cultures per point. 


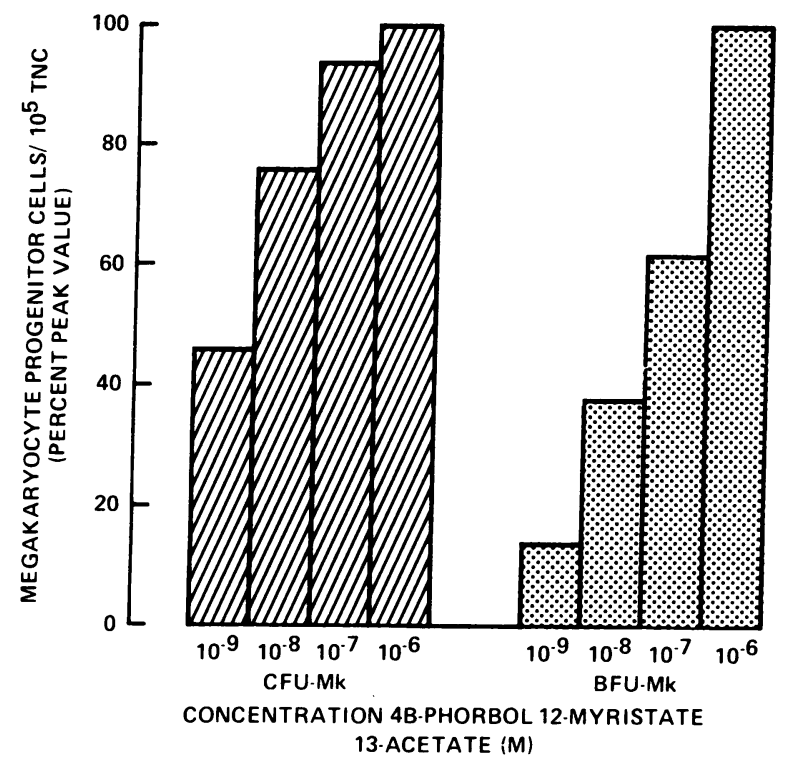

Figure 5. Responsiveness of Mk progenitor cells to PMA. Mk progenitor cells were cultured in the presence of WEHI-3 CM (5\%) and varying concentrations of PMA. In order to compare responsiveness of these two progenitor cells, the numbers of colonies cultured were normalized as percent of peak value. Separate assays performed at limiting dilutions for each progenitor cell (i.e., $75,000 \mathrm{cells} / \mathrm{ml}$ for CFU-Mk and 33,000 cells/ml for BFU-Mk). $n=5$ experiments, except for $10^{-9}$ and $10^{-8}$ M PMA in the BFU-Mk, which is three experiments. Peak values are CFU-Mk, 29.0 \pm 6.6 ; BFU-Mk, 7.3 \pm 0.7 per $10^{5} \mathrm{TNC}$.

(WEHI-3 CM) over a range of $10^{-9}-10^{-6} \mathrm{M}$. This was true for both CFU-Mk and BFU-Mk. However, the BFU-Mk required a tenfold greater concentration of PMA for optimal colony development. BFU-Mk were observed in low numbers $(3.0 \pm 2.0$ / $10^{5}$ TNC) in cultures containing WEHI-3 CM and MLuCM.

It is possible that phorbol diesters affect $\mathbf{M k}$ progenitor cells indirectly via known accessory cell populations $(11,12)$. However, we have shown that such an indirect effect is unlikely (10). Moreover, recent work in this laboratory shows that murine Mk progenitor cells exhaustively depleted of immunoregulatory cells ( $T$ cells, B cells, and macrophages) still respond to PMA (Long, M. W., unpublished observations). Moreover, PMA-responsive BFU-Mk and CFU-Mk are observed in cultures of highly purified stem cell populations ( $\simeq 30 \% \mathrm{CFU}$ ) when plated at 250 cells/ml - a density at which residual accessory cells (if any) would be ineffective (unpublished observations).

Kinetic analysis of $M k$ colony development. The progenitorprogeny relationship between BFU-Mk and CFU-Mk was examined in sequential studies of $\mathrm{Mk}$ colony development (Fig. 6). The CFU-Mk required 5-7 d for the detection of optimal numbers of progenitor cell colonies. The BFU-Mk, however, did not show optimal development until 10-12 d in culture. These two progenitor cells also showed distinctly different developmental patterns in the early phases of culture. In the first $5 \mathrm{~d}$ of culture, the BFU-Mk generated only $35 \%$ of the total number of colonies, whereas the CFU-Mk generated $\sim 80 \%$ of optimal numbers.

Physical characteristics of Mk progenitor cells. Mk progenitor cells also differed in their physical characteristics. Velocity sedimentation profiles are shown in Fig. 7. The BFU-Mk had

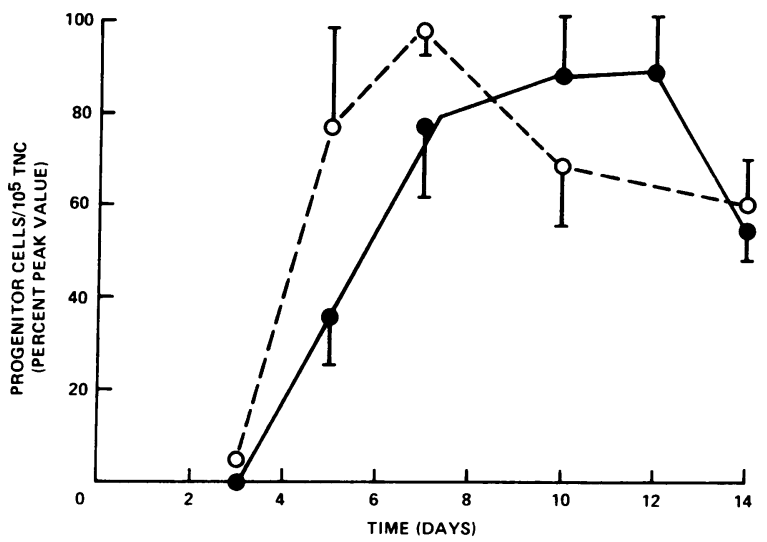

Figure 6. Kinetics of Mk progenitor cell development. Mk progenitor cells were cultured at limiting dilutions (as in Fig. 5) in the presence of WEHI-3 CM and $10^{-6}$ M PMA. Solid circles, BFU-Mk; open circles, CFU-Mk. Values are $\mathrm{X} \pm \mathrm{SD}$ of four experiments of 3-5 replicate cultures per point. Peak values as in Fig. 5.

a restricted velocity sedimentation profile compared with that of the CFU-Mk, sedimenting over a range of $3.3-5.3 \mathrm{mmh}^{-1}$ compared with 3.4-7.5 $\mathrm{mmh}^{-1}$, respectively. The CFU-Mk profile also contained a pronounced shoulder occurring between 5.0 and $7.0 \mathrm{mmh}^{-1}$, which was absent in the BFU-Mk curve. Mixed colonies (i.e., ACh-E positive and negative cells within a single colony) were not seen to distribute according to their sedimentation velocity (data not shown). The equilibrium density distribution of $\mathrm{Mk}$ progenitor cells ranged from 1.058 to $1.080 \mathrm{~g} / \mathrm{cm}^{3}$ for the BFU-Mk and $1.063-1.080 \mathrm{~g} / \mathrm{cm}^{3}$ for CFU-Mk (Fig. 8, top). Rebanding experiments indicated both progenitor cells were achieving equilibrium density (Fig. 8, middle). The modal density of the BFU-Mk was observed to be consistently lower than the CFU-Mk $(1.068 \pm 0.0002$ vs. $1.070 \pm 0.002$, respectively; Fig. 8 , bottom). However, the variabilities of the modal buoyant densities were less than the limits of resolution of this system, implying that the two

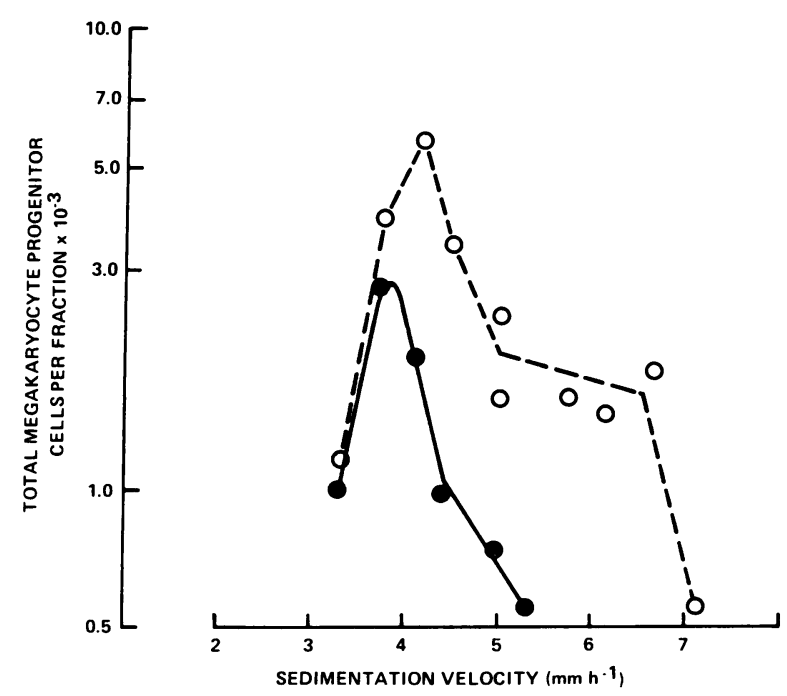

Figure 7. Velocity sedimentation characteristics of $\mathrm{Mk}$ progenitor cells. Open circles, CFU-Mk; closed circles, BFU-Mk. A single representative experiment of four separate velocity sedimentations is shown. Colonies cultured as in Fig. 6. 


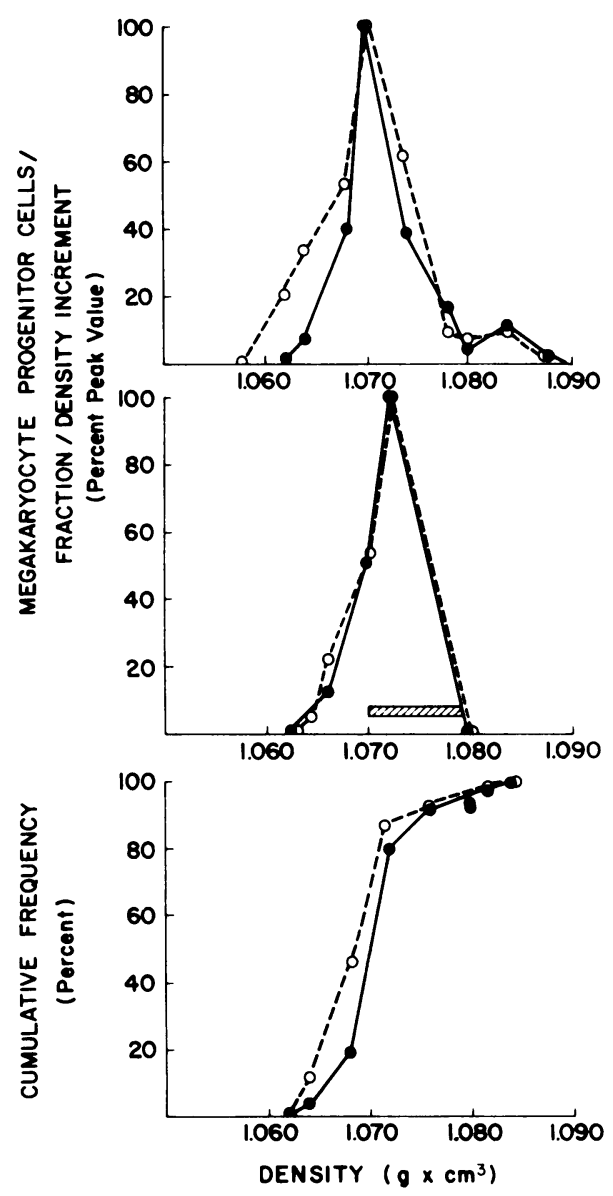

Figure 8. Buoyant density characteristics of murine Mk progenitor cells. Open circles, BFU-Mk; closed circles, CFU-Mk. (Top) Distribution of progenitor cell buoyant densities. (Middle) Density distribution of progenitor cells subjected to a second density centrifugation; hatch bar shows density range of progenitor cells in first gradient, which were loaded onto the second gradient. (Bottom) Modal buoyant densities determined by plot of cumulative frequencies. Profiles (top, middle) from single, separate density centrifugations of 3-5 replicate cultures per point. Values (bottom) are means of four separate density gradients of 3-5 replicate cultures per density fraction per experiment. Colonies cultured as in Fig. 6. Values (bottom) are means of four separate density gradients of 3-5 replicate cultures per density fraction per experiment.

progenitor cells cannot be separated by equilibrium density centrifugation.

Proliferative potential of $M k$ progenitor cells. The proliferative potential of these two progenitor cells differed greatly during colony development (Table I). Within $5 \mathrm{~d}$ of culture, the CFU-Mk generated some 16.5 \pm 1.2 (mean \pm SEM) Mk/ colony. No further significant increase in colony size was seen throughout the subsequent $9 \mathrm{~d}$ of culture. The BFU-Mk generated four times as many Mk per burst within $5 \mathrm{~d}$, and reached levels of $121 \pm 11 \mathrm{Mk}$ by day 14 of culture-representing an 8.6-fold increase over the cellularity of the CFU-Mk. Moreover, analysis of the number of cells per colony within a burst (i.e., the number of cells per foci within a burst) showed that the individual BFU-Mk subcolonies contained approximately two times as many Mk as did the CFU-Mk.

Lineage-restriction of $\mathrm{Mk}$ progenitor cells. In order to
Table I. Kinetic Analysis of Progenitor Cell Colony Cellularity

\begin{tabular}{|c|c|c|c|c|}
\hline \multirow[b]{2}{*}{ Day } & \multirow{2}{*}{$\frac{\text { CFU-Mk }}{\text { Cells/colony }}$} & \multicolumn{2}{|l|}{ BFU-Mk } & \\
\hline & & Cells/colony & Total cells/burst & \\
\hline & & & & $n$ \\
\hline 5 & $16.5 \pm 1.2$ & $20.5 \pm 2.1$ & $70.8 \pm 9.0^{*}$ & $167 / 81$ \\
\hline 7 & $17.2 \pm 1.3$ & $24.9 \pm 1.2^{*}$ & $82.4 \pm 5.6^{*}$ & $246 / 223$ \\
\hline 10 & $19.3 \pm 1.5$ & $30.7 \pm 1.5^{*}$ & $118.5 \pm 8.8^{*}$ & $254 / 163$ \\
\hline 12 & $19.0 \pm 2.4$ & $30.9 \pm 1.5^{*}$ & $116.1 \pm 8.3^{*}$ & $94 / 144$ \\
\hline 14 & $14.0 \pm 1.5$ & $37.0 \pm 2.1^{*}$ & $121.0 \pm 11.0^{*}$ & $49 / 36$ \\
\hline
\end{tabular}

Values are $\mathrm{X} \pm \mathrm{SEM}$. $n$, Number of BFU-Mk and CFU-Mk colonies counted, respectively.

${ }^{*} P \leq 0.05$ (comparison is vs. CFU-Mk cellularity for a given day).

examine whether early Mk progenitor cells were restricted to this lineage, both BFU-Mk and CFU-Mk were cultured in the presence of WEHI-3 CM (7.5\%), $10^{-6} \mathrm{M}$ PMA, and various concentrations of both sheep and murine erythropoietin $(0.025$, $0.05,1.0$, and $2.0 \mathrm{U} / \mathrm{ml}$ ) in plasma clot cultures. Parallel cultures were stained with benzidine-for hemoglobin content or ACh-E-a specific cytochemical marker for murine Mk. As mentioned, the ACh-E reaction indicated that $90 \%$ of the colonies were comprised entirely of $\mathrm{ACh}-\mathrm{E}$-containing cells. Benzidine-positive cells were not observed in Mk colonies formed in parallel cultures (colonies identified in benzidinestained cultures by their morphology, as both reaction products appear yellow-brown). Both partially purified sheep erythropoietin (Connaught step III) and murine erythropoietin (HyClone Inc.) gave similar results, as did agar-based cultures (data not shown). Finally, the $10 \%$ of the mixed $\mathrm{ACh}-\mathrm{E}$ positive and negative colonies did not distribute according to their sedimentation velocity characteristic, indicating that these were coincidental colonies even at the limiting dilution of 3.3 $\times 10^{4} \mathrm{TNC} / \mathrm{ml}$.

Quantitation of murine femoral progenitor cell content. The Mk progenitor cell compartment in the femur was examined in a series of individual mice (Table II). There are $\sim 1,000$ BFU-Mk/femur in these animals, compared with $\sim 4,600$ CFU-Mk. This increases the size of known Mk progenitor cell compartment by $20 \%$ over reported values (17).

\section{Discussion}

This communication presents evidence for the existence of a new Mk progenitor cell, the BFU-Mk. Morphologically, Mk bursts are comprised of 2-7 subcolonies containing $\sim 40-500$ $\mathrm{ACh}-\mathrm{E}$-positive Mk. The in vitro development of the burstforming progenitor cells requires two synergistic activities for

Table II. Femoral Content of Murine Mk Progenitor Cells

\begin{tabular}{lcc}
\hline & CFU-Mk & BFU-Mk \\
\hline Frequency per $10^{5}$ TNC & $36.7 \pm 2.5$ & $7.3 \pm 0.7$ \\
Total progenitor cells per femur & $4,632 \pm 317$ & $936 \pm 86$ \\
\hline
\end{tabular}

Values are mean \pm SEM for 27 animals based on 3-5 replicate cultures per animal. The total marrow cellularity was $139 \pm 9.0 \times 10^{5}$ TNC/femur. 
development, shows a decreased sensitivity to PMA, and has delayed in vitro growth patterns. Finally, these early Mk progenitor cells have an increased proliferative potential as represented by their total colony cellularity and the cellularity of each individual colony (or focus) within a burst.

The BFU-Mk represent the earliest cells detected in the Mk lineage. This is shown by their high degree of proliferative potential, altered responsiveness to stimuli, and delayed in vitro development. Hematopoietic stem cells are characterized functionally by criteria such as in vitro development, proliferative potential, and responsiveness to stimuli (18). Such developmental differences, along with physical properties, define the position of a given progenitor cell within a maturation sequence. Thus, progenitor cells early in the differentiation process should be less dense, sediment more slowly, and be capable of generating larger numbers of differentiated progeny than later committed progenitor cells. All of these are properties of the BFU-Mk. Similar characteristics are observed in the granulocytic HPP-CFC and in the BFU-e (1-4).

Mk bursts were not detected in previous reports of in vitro Mk colony development (5-9). However, Williams and Jackson (19) demonstrated that Mk progenitor cells (i.e., the CFU-Mk) are heterogeneous with respect to their proliferative, differentiative, and endoreduplicative potentials. Indeed, all the in vitro developmental characteristics reported by Williams and Jackson (19) for the CFU-Mk are similar to the characteristics reported for the CFU-Mk in this manuscript. Thus, their frequency analysis of the number of Mk per colony indicate that $14 \%$ of the CFU-Mk have $\geq 41$ cells, whereas we find that $11 \%$ of the CFU-Mk contain this number of cells. Also, the bone marrow incidence, factor requirements, kinetics of in vitro development, and colony cellularity of the CFU-Mk presented in these two papers are nearly identical. Levin et al. (8) reported two morphologically distinct classifications of murine CFU-Mk. He observed murine Mk colonies that are restricted in the number of cells per colony ( $\simeq 16$ cells) and have high DNA content, as well as larger heterogenous colonies ( $\simeq 44$ cells) of lower ploidy values that are comprised of both small and large Mk. Neither of these reports show colonies of the morphological or in vitro developmental characteristics of the BFU-Mk.

Recently, Thean et al. (20) reported the presence of an early Mk progenitor cell in an in vivo assay system. These investigators detected the presence of an early Mk precursor cell (termed the Mk-CFU spleen [Mk-CFUs]) in the spleens of lethally irradiated recipients. These investigators suggest that these progenitor cells (after treatment with 5-fluorouracil) have a higher proliferative potential than such cells detected in normal bone marrow. However, the relationship between the Mk-CFUs and known Mk progenitor cells (CFU-Mk) is unknown (20), as is the relationship between the BFU-Mk and the Mk-CFUs.

The physical characteristics of the BFU-Mk show that these cells are among the lightest and/or smallest of the Mk progenitor cells, and are not comprised of clumps of CFUMk. Were the latter true, BFU-Mk would sediment at multiples of the CFU-Mk sedimentation rate (i.e., multiples of 4-5 $\mathrm{mmh}^{-1}$ ). The lack of significant physical differences between the CFU-Mk and BFU-Mk is not surprising, as hematopoietic progenitor cells have very similar physical characteristics. The CFU macrophage, CFUe, CFU-Mk, and BFUe have peak sedimentation rates of $4.0-4.8 \mathrm{mmh}^{-1}(21-26)$. Modal buoyant densities (for type $1 \mathrm{CFU}$ granulocyte/macrophage, CFU-Mk, CFU spleen, and HPP-CFC) are $1.070 \mathrm{~g} / \mathrm{cm}^{3}(4,21-26)$. These values are similar to those presented in this report for $\mathbf{M k}$ progenitor cells.

The restriction of both the BFU-Mk and CFU-Mk to the Mk lineage is explicit in the observation that $90 \%$ of the former and $100 \%$ of the latter contain only ACh-E positive cells. ACh-E is a specific cytochemical marker of murine Mk (27). Moreover, the few mixed colonies (i.e., ACh-E positive and negative cells within a single colony) observed do not distribute according to a known physical characteristic (their sedimentation velocity), which suggests that these are coincidental colonies. Finally, the lack of erythropoietin responsiveness in these progenitor cells is further evidence of their lineage restriction. However, it should be noted that PMA has been reported to inhibit erythroid colony formation $(28,29)$. Its presence may thus mask the actions of erythropoietin in these cultures. We conclude that $90 \%$ of the BFU-Mk are restricted to the Mk lineage. Whether the remaining $10 \%$ of the BFUMk are truly of mixed cellularity (i.e., stemming from a multipotent progenitor cell) or coincidental colonies must await confirmation by clone-transfer experiments.

In this communication, we report the presence of a newly described murine progenitor cell, BFU-Mk, which is characterized by high proliferative potential, altered sensitivity to in vitro regulatory factors, and prolonged in vitro development. These observations support the hypothesis that these cells are early Mk precursor cells which antedate the CFU-Mk. As well, these data extend and strengthen similar observations showing that the myeloid lineages (i.e., granulocytic, erythrocyte, and now, megakaryocytic) contain cells very early in the differentiation process that are restricted (or committed) to a given lineage but retain a high degree of proliferative potential.

\section{Acknowledgments}

The authors are indebted to Anne Busch for excellent preparation of the manuscript.

This work was supported in part by grants HL31568 from the National Institutes of Health and 83-1169 from the American Heart Association.

\section{References}

1. Iscove, N. N., and F. Sieber. 1975. Erythroid progenitors in mouse bone marrow detected by macroscopic colony formation in culture. Exp. Hemat. (Lawerence). 3:32-43.

2. Iscove, N. N., F. Sieber, and K. H. Winterhalter. 1974. Erythroid colony formation in mouse and human bone marrow: analysis of the requirement for erythropoietin by gel filtration and affinity chromatography on Agarose-concanavalin A. J. Cell. Physiol. 83:309-320.

3. Bradley, T. R., and G. S. Hodgson. 1979. Detection of primative macrophage progenitor cells in mouse bone marrow. Blood. 54:14461450.

4. Bains, P., S. Bol, and M. Rosendaal. 1981. Characterization of a developmentally early macrophage progenitor found in normal mouse marrow. Br. J. Haematol. 48:147-153.

5. Metcalf, D., H. R. McDonald, N. Odartchenko, and B. Sordat. 1975. Growth of mouse megakaryocyte colonies in vitro. Proc. Natl. Acad. Sci. USA. 73:1744-1748.

6. Williams, N., and H. Jackson. 1978. Regulation of the proliferation of murine megakaryocyte progenitor cells by cell cycle. Blood. 52:163-169.

7. Burstein, S., J. W. Adamson, D. Thoring, and L. A. Harker. 
1979. Characteristics of murine megakaryocyte colonies in vitro. Blood. 54:169-179.

8. Levin, J., F. C. Levin, D. G. Penington, and D. Metcalf. 1981 Measurement of ploidy distribution in megakaryocyte colonies obtained from culture: with studies of the effects of thrombocytopenia. Blood. 57:287-297.

9. Nakeff, A., K. A. Dicke, and M. J. vanNoord. 1975. Megakaryocytes in agar cultures of mouse bone marrow. Ser. Hematol. 8:421.

10. Long, M. W., J. E. Smolen, P. Szczepanski, and L. A. Boxer. 1984. Role of phorbol diesters in in vitro murine megakaryocyte colony formation. J. Clin. Invest. 74:1686-1692.

11. Williams, N., R. R. Eger, H. M. Jackson, and D. J. Nelson. 1982. Two factor requirement for murine megakaryocyte colony formation. J. Cell Physiol. 110:101-104.

12. Williams, N., H. M. Jackson, R. R. Eger, and M. W. Long. 1981. The separate roles of factors in murine megakaryocyte colony formation. In Megakaryocytes In Vitro: Biology and Precursors. B. Evatt, R. Levine, and N. Williams, editors. Elsevier Science Publishing Co., Inc., New York. 59-75.

13. Ralph, P., M. A. S. Moore, and K. Nilsson. 1976. Lysosyme synthesis by established human and murine histocyte lymphoma cell lines. J. Exp. Med. 143:1528-1533.

14. Long, M. W., and N. Williams. 1982. Differences in the regulation of megakaryocytopoiesis in the murine bone marrow and spleen. Leukemia Research. 6:721-728.

15. Miller, R. G., and R. A. Phillips. 1969. Separation of cells by velocity sedimentation. J. Cell Physiol. 73:191-202.

16. Bol, S., J. Visser, N. Williams, G. vandenEngh. 1977. Physical characterization of hematopoietic progenitor cells by equilibrium density centrifugation. In Cell Separation Methods. H. Bloemendal, editor. Elsevier/North Holland Biomedical Press, Amsterdam, The Netherlands. 39-52.

17. Long, M. W., and N. Williams. 1981. Immature megakaryocytes in the mouse: morphology and quantitation by acetylcholinesterase staining. Blood. 58:1032-1039.

18. Rosendaal, M., G. S. Hodgson, and T. R. Bradley. 1979. Organization of haemopoietic stem cells: the generation-age hypothesis. Cell Tissue Kinet. 12:17-29.
19. Williams, N., and H. Jackson. 1982. Kinetic analysis of megakaryocyte numbers of ploidy levels in developing colonies from mouse bone marrow cells. Cell Tissue Kinet. 15:483-494.

20. Thean, L. E., G. S. Hodgson, I. Bentoncello, and J. M. Radley. 1983. Characterization of megakaryocyte-spleen colony forming units by response to 5 -flurouracil and by unit-gravity sedimentation. Blood. 62:896-901.

21. Metcalf, D., H. R. MacDonald, N. Odartchenko, and B. Sardat. 1975. Growth of mouse megakaryocyte colonies in vitro. Proc. Natl. Acad. Sci. USA. 72:1744-1748.

22. Metcalf, D., and H. R. MacDonald. 1975. Heterogeneity of in vitro colony and cluster-forming cells in the mouse marrow: segregation by velocity sedimentation. J. Cell Physiol. 85:643-654.

23. McVitte, T. J., and K. F. McCarthy. 1977. The detection of in vitro monocyte-macrophage colony forming cells in mouse thymus and lymph nodes. J. Cell Physiol. 92:203-207.

24. Bol, S., J. Visser, and G. vandenEngh. 1977. The physical separation of three subpopulations of granulocyte/macrophage progenitor cells from mouse bone marrow. Exp. Hemat. (Lawerence). 7:541553.

25. Wagemaker, G., M. F. Peters, and S. J. Bol. 1979. Induction of erythropoietin responsiveness in vitro to a distinct population of bone marrow cells. Cell Tissue Kinet. 12:521-537.

26. Williams, N., H. Jackson, and P. Meyers. 1979. Isolation of pluripotent hemopoietic stem cells and clonable precursor cells of erythrocytes, granulocytes, macrophages and megakaryocytes from mouse bone marrow. Exp. Hemat. (Lawerence). 7:524-534.

27. Jackson, C. W. 1973. Cholinesterase as a possible marker of early cells of the megakaryocyte lineage. Blood. 42:413-421.

28. Sieber, F., R. K. Stuart, and J. L. Spivak. 1981. Tumorpromoting phorbol esters stimulate myelopoiesis and suppress erythropoiesis in cultures of mouse bone marrow cells. Proc. Nat. Acad. Sci. USA. 78:4402-4406.

29. Fibach, E., R. Bambari, P. A. Shaw, G. Maniatis, R. C. Ruben, S. Sassa, R. A. Rivkind, and P. A. Marks. 1979. Tumor-promptor. Mediated inhibition of cell differentiation: suppression of the expression of erythroid function in murine erythroleukemia cells. Proc. Nat. Acad. Sci. USA. 76:1906-1910. 